\title{
Transatlantica
}

Revue d'études américaines. American Studies Journal

Paule Lévy, Figures de l'artiste, Identité et écriture dans la littérature juive américaine de la deuxième moitié du XXe siècle. Pessac, Presses universitaires de Bordeaux, 2006. 191 pages. (ISBN $10: 2867813913$, ISBN 13 : 9782867813917 ).

\section{Emmanuel Vernadakis}

\section{OpenEdition}

Édition électronique

URL : https://journals.openedition.org/transatlantica/4720

DOI : $10.4000 /$ transatlantica.4720

ISSN : 1765-2766

Éditeur

Association française d'Etudes Américaines (AFEA)

Référence électronique

Emmanuel Vernadakis, «Paule Lévy, Figures de l'artiste, Identité et écriture dans la littérature juive américaine de la deuxième moitié du XXe siècle. Pessac, Presses universitaires de Bordeaux, 2006. 191 pages. (ISBN 10 : 286781391 3, ISBN 13 : 978286781391 7). », Transatlantica [En ligne], 2 | 2009, mis en ligne le 02 février 2010, consulté le 01 février 2023. URL : http://journals.openedition.org/ transatlantica/4720 ; DOI : https://doi.org/10.4000/transatlantica.4720

Ce document a été généré automatiquement le 1 février 2023

Creative Commons - Attribution - Pas d'Utilisation Commerciale - Pas de Modification 4.0 International - CC BY-NC-ND 4.0

https://creativecommons.org/licenses/by-nc-nd/4.0/ 


\title{
Paule Lévy, Figures de l'artiste, Identité et écriture dans la littérature juive américaine de la deuxième moitié du XXe siècle. Pessac, Presses universitaires de Bordeaux, 2006. 191 pages. (ISBN $10: 2867813913$, ISBN 13 : 9782867813917 ).
}

\author{
Emmanuel Vernadakis
}

1 L'ouvrage de Paule Lévy, Figures de l'artiste, Identité et écriture dans la littérature juive américaine de la deuxième moitié du XXe siècle, est le troisième volume paru dans la collection «Lettres d'Amérique(s) », dirigée par Yves-Charles Grandjeat ${ }^{1}$, aux Presses Universitaires de Bordeaux. Le corpus de ce nouvel ouvrage se constitue d'une dizaine de romans, nouvelles et novellas réflexives dans lesquelles l'auteur étudie la représentation littéraire des interactions culturelles dans la construction, déconstruction et reconstruction identitaires de sept auteurs: Saul Bellow, Bernard Malamud, Philip Roth, Cynthia Ozick, Grace Paley, Tillie Olsen et Henry Roth. L'ouvrage est de deux cents pages et se compose d'une introduction, de sept chapitres consacrés respectivement à chacun des auteurs sus-mentionnés, d'une conclusion et d'une bibliographie.

2 Dans son introduction (p. 11-22), l'auteur présente un historique concis de la littérature juive américaine en rappelant que, par-delà un bagage culturel et religieux commun, la diversité des langues, des traditions, des destins et des sensibilités au sein du monde juif nous commande d'évoquer cette littérature au pluriel. Difficile à définir, celle-ci connaît dans les années soixante, soixante-dix une nouvelle période de développement marquée, curieusement, par l'annonce de sa mort $^{2}$. Celle-ci n'adviendra pas. Il n'en fut 
rien cependant. La littérature juive américaine trouve d'autres lieux d'ancrage pour se renouveler : « La dialectique de l'ici et de l'ailleurs [...], si marquée dans les productions juives américaines antérieures » (p. 13-14) fait progressivement place à une remise en cause des formes traditionnelles et à un questionnement d'ordre éthique, esthétique et existentiel. "Réels ou fantasmés ", ces lieux d'ancrage, pour nouveaux qu'ils soient, s'inscrivent dans tout un processus et une tradition très ancienne du questionnement qui remonte à la Bible et marque jusqu'aux oeuvres littéraires de la fin du siècle dernier. Comme le montre Paule Lévy, l'interrogation est intrinsèquement liée à l'écriture juive.

3 En guise de préalable, l'auteur pose, elle aussi, une série de questions qui reflètent son corpus : «Qu'est-ce au juste qu'un écrivain juif ? Comment, pour qui et sur quoi doit-il écrire?» (p.14); "À concevoir le Juif en termes génériques ne risque-t-on pas de tomber dans des stéréotypes réducteurs (positifs ou négatifs) qui lui sont associés [...] ? »; « À penser le Juif d'un seul bloc n'occulte-t-on pas les conflits qui agitent tant l'individu singulier que le groupe dans son ensemble ?»; « ...la recherche de critères définitoires [...] n'implique-t-elle pas une conception essentialiste et datée de l'identité [...] à l'heure où [l'identité est conçue] comme un processus, une construction dépendante du contexte où elle s'inscrit? "; "Qu'est-ce qu'un Américain et comment se distingue-t-il d'un Européen?» (p. 15); « the Jewish question, the Woman question-Who is the questioner? Who is supposed to answer ?" " (p. 20). Puis, "Comment concilier le particulier et l'universel, comment s'inscrire dans le cœur des voix américaines sans pour autant se détourner de la culture juive ? De cette culture que reste-t-il, au juste, à l'heure où la Shoah, puis l'assimilation ont sapé les fondements sociaux et linguistiques de la vie juive [...], à l'heure enfin où la création de l'État d'Israël a radicalement modifié les mentalités juives et non juives?»(p. 21). L'ouvrage tient toutes les promesses du titre par le biais d'un jeu de questions issues des œuvres d'un corpus dont les auteurs "se connaissent [...] s'observent [...] se lisent et, [...] se critiquent mutuellement" (p. 16). Par la variété des points de vue de ceux qui les posent, les questions conduisent à une compréhension plus fine de leur objet, mais aussi du sujet - du livre - qu'elles introduisent: l'ouvrage se penche sur une littérature qui finit "par faire du questionnement son unique vérité et du doute son unique sagesse » (p. 22) afin de nous offrir une connaissance approfondie de la pluralité à la fois américaine et juive, au sein de l'identité juive américaine.

Consacré à la relation de l'individu à l'espace et au temps, l'ouvrage aborde la question de l'identité perçue en termes de continuité et de rupture. Cette problématique est ici abordée à la lumière de la théorie et de la critique actuelles. Mikhail Bakhtine, Roland Barthes, Jean Baudrillard, Jacques Derrida, Maurice Blanchot, Philippe Hamon, Harold Bloom, Frank Kermode, Gilles Deleuze, Gérard Genette, Julia Kristeva, Daniel Sibony, Georges Steiner ou Tzvetan Todorov sont convoqués au cours de l'analyse, tout comme les textes de Freud, Lacan, Assoun, Anzien, Green et Bellamy ou encore ceux de Finkielkraut, de Lévinas et de Ricoeur. L'auteur fait également appel aux travaux des spécialistes de la littérature juive américaine tels que Robert Alter, Martine ChardHutchinson, Rachel Ertel, Pierre-Yves Pétillon, Ada Savin, Suzanne Durruty, Judith Stora-Sandor entre autres. Les cinq romans, trois nouvelles longues (novellas) et deux brèves (short stories) du corpus sont réflexifs de par leur thématique, leur structure narrative ou les deux. Les personnages principaux y sont des écrivains en devenir (Henderson the Rain King de Bellow), en quête de filiation (The Ghost Writer de Roth), en peine d'inspiration (The Tenants de Malamud), des écrivains confirmés (Humboldt's Gift 
de Bellow), ou encore des narratrices en quête de narration («Usurpation » d'Ozick) des narratrices qui s'effacent ("Tell me a Riddle» d'olsen) ou sont en rupture par rapport à la tradition narrative ( A Conversation with my Father » de Paley). Classées chronologiquement en fonction des dates de parution des œuvres, les études se penchent sur « le statut de l'écrivain juif américain, sur ses modes d'expression et sur la portée de son œuvre» (p.16) afin de faire émerger « des lignes de sens, des échos ou des dissonances » qui attestent à la fois « de la cohésion et de la diversité » (p. 22) de la littérature juive américaine. Notons toutefois, que chaque chapitre peut, le cas échéant, faire l'objet d'une lecture séparée.

La façon de procéder de l'auteur est sensiblement la même d'un chapitre à l'autre. Formé du nom de l'écrivain étudié, chaque titre est suivi d'un sous-titre qui, par le biais d'une figure de style ou d'une réécriture décalée, circonscrit de manière poétique ce que Paule Lévy suggère être la spécificité de cet écrivain (ex. "Grace Paley: Rires et chuchotements ", « Henry Roth : l'Art de dire l'indicible »). Chaque étude est introduite par un exposé sommaire et critique de la carrière de l'écrivain étudié, mise en perspective par une ou plusieurs citations. Il est suivi d'une section consacrée à l'analyse d'une œuvre individuelle ${ }^{4}$ qui commence par un résumé de l'intrigue et qui se divise ensuite en plusieurs sous-sections, elles aussi pourvues de titres individuels de facture poétique, ainsi que d'épigraphes. Dans les sous-sections, la forme métafictionnelle de chaque œuvre, son formalisme si l'on préfère, est expliqué(e) dans son rapport au sens à la lumière des théoriciens et critiques sus-mentionnés, au moyen de citations commentées et de micro-lectures aboutissant à des synthèses, bilans d'étape, macro-lectures et conclusions. Chaque œuvre se voit ainsi méthodiquement étudiée, avec inspiration, générosité, prudence, précision, et honnêteté. L'analyse s'arrête lorsque les titres et sous-titres se trouvent explicités, lorsque l'enjeu poétique de la figure de style ou de la réécriture littéraire qui les constituent parvient à signifier sur un plan littéral. Les citations en exergue s'avèrent, elles aussi, jouer un rôle dynamique dans la construction du sens. Après la lecture de chaque chapitre, un retour aux titres et aux citations en exergue se révèle gratifiant parce qu'ils fonctionnent alors comme un précis de ce que le lecteur a lu.

6 Prenons pour exemple le premier chapitre (p. 25-50), intitulé «L'écriture en suspens ", consacré au prix Nobel de littérature (1975) Saul Bellow (1915-2005), dont deux romans sont étudiés en particulier: Henderson, the Rain King (1959) et Humboldt's Gift (1975). L'étude commence par une présentation critique de la carrière de Bellow (p. 25-26) que trois citations en exergue mettent en perspective.

Aux saules de la contrée,

Nous avions suspendu nos harpes (...)

Comment chanterions- nous

Les cantiques de l'Éternel

Sur une terre étrangère?

Psaume 137

Son cœur est un luth suspendu

Sitôt qu'on le touche, il résonne.

Épigraphe à « The Fall of the House of Usher » attribuée à Béranger par Edgar Allan

Poe

It's one of these luths suspendus deals, sitôt qu'on le touche, il résonne.

Saul Bellow, Henderson, the Rain King

7 Le participe passé « suspendu », qui se retrouve dans les trois citations, renvoie au titre «L'écriture en suspens ». Par ailleurs, on remarque que celles-ci se rattachent les unes 
aux autres par le thème de la musicalité présent dans les trois. On présume que leur fonction est d'annoncer le biais par lequel l'étude abordera la thématique identitaire dans le contexte métafictionnel des œuvres étudiées. Henderson, the Rain King, est examiné dans une première section, elle-même divisée en quatre sous-sections ( Henderson, the Rain King : roman du rugissement et de l'échappée belle », « Le poids de l'héritage, les cassures de la filiation ", "En quête de l'innocence absolue », "La richesse du mélange », p. 27-38). Il en est de même pour Humboldt's Gift, étudié, quant à lui, dans cinq sous-sections (« Humboldt's Gift : Entre l'ici etl'ailleurs », « La rançon de la gloire », «L'artiste chez les cannibales », « La patrie perdue des origines », « Morale et écriture : un art du compromis ", p. 38-51).

8 Au cours de l'étude, Henderson, the Rain King, roman qui s'articule autour du rapport filial à « l'héritage paternel », s'avère être un mélange d'épopée et de farce (p. 27) entre deux traditions. Paule Lévy y décèle une écriture plurielle en quête d'elle-même et de quelques modes spécifiques qu'elle parvient à isoler pour nous les exposer tour à tour. L'écriture est faite de citations, allusions et reprises de toutes sortes qui "sont si nombreuses qu'il serait impossible de les répertorier [...] » (p. 34). À la fois introspective et picaresque, elle met en évidence «la faillite du matérialisme, cet autre aspect du rêve américain qui est toujours chez Saul Bellow synonyme de dénuement spirituel » (p. 29). La pluralité de cette écriture renvoie à la nature hétérogène de l'héritage à la fois juif et américain de Bellow. Identifié dans la facture de l'écriture, cet héritage se reflète aussi dans la création du protagoniste du roman: Eugene Henderson, héritier d'un père écrivain et pourvu d'« un moi avide de se soustraire aux modèles imposés mais incapable de se reconnaître dans son intégrité [...], [est] un agglomérat d'éléments disparates» (p. 31). Bellow en fait un aventurier, solitaire et viril, qui entreprend une quête initiatique, de manière à ce que l'on reconnaisse dans ce personnage les traits du héros hemingwayen. Ces traits se marient néanmoins avec ceux du shlemiehl, personnage typique de la littérature yiddish qui constitue une des principales sources du comique dans cette tradition. En réalité donc, l'agglomérat d'éléments disparates qui constituent le personnage de Henderson reflète le fruit d'un métissage littéraire qui n'a peut-être pas encore mûri. Mais revenons à l'écriture plurielle et burlesque dont Paule Lévy montre qu'elle est également affiliée à la tradition biblico-talmudique de l'exégèse circulaire "fondée sur une constante confrontation des points de vue " (p.35). Le principe du métissage littéraire se constate donc aussi sur le plan des traditions, des registres et des genres. Le décalage qui en résulte est source de comique et donne au roman et à son protagoniste une solidité qui leur est propre.

[chaque citation, allusion, référence ou reprise] participe au comique par le décalage qu'elle induit. Mais c'est dans leur foisonnement, dans leur ironique orchestration, dans le mélange des cultures, des registres, et des genres que réside la force de ce texte qui se révèle à l'image du héros: [...] multiforme [...] et plurivoque : 'It's one of those luths suspendus deals, sitôt qu'on le touche, il résonne.' (p. 35)

9 De manière subtile, le thème de la parodie, dont le sens étymologique est «chanter avec un décalage ", est ici introduit en mettant en contexte la référence de Bellow à Poe citée en épigraphe au début du chapitre. E. A. Poe est convoqué pour participer à la transformation de la traversée initiatique de Henderson, qui est en quête d'innocence, en "traversée de l'écriture» (p. 35): «Some Passages in the Life of a Lion» ou «Lionising " est le titre d'une nouvelle de Poe dont le protagoniste réussit à se faire admettre dans une famille littéraire, « en lion ». C'est le même sort que Bellow réserve 
symboliquement à Henderson qui, pour ressembler à son frère aîné, « un vrai lion » qui «mourut pour une absurde histoire de stylo et de lettre » (p. 35) se métamorphosera lui aussi en lion par le biais de la langue («lion»: A prominent person who is in demand socially. A celebrity). «Devenir 'lion' pour Henderson, c'est en fait acquérir les lettres de noblesse qui lui permettront de succéder dignement à son illustre père, en prenant la plume à son tour pour conter ses aventures » (p. 35).

L'objectif des trois citations mises en épigraphe devient alors explicite. Les « harpes» du psaume sont, comme les luths de Poe, comme Saul Bellow et les écrivains juifs américains de Paule Lévy, à même d'accompagner les chants de l'Éternel même en terre étrangère ; toutefois ils le feront par le biais de la parodie qui les rend particulièrement sensibles au toucher : aussitôt qu'on les touche, elles résonnent. "La parodie, écrit Paule Lévy, permet d'établir une forme d'harmonie en marquant un écart [...] elle constitue un moyen de reconnaître une filiation avec les modèles mêmes qu'on ébranle» (p. 36). Au lieu de traverser des paradis terrestres inconnus, Henderson traverse ainsi l'écriture des autres pour nous montrer que, comme l'indique Paule Lévy: "Il n'est point d'innocence, d'absolu ou de territoire vierge, et le plus beau continent à explorer c'est l'Autre » (p. 36).

Intitulé "Locataire dans la "maison d'écriture" " le deuxième chapitre (p. 53-66) est consacré à Bernard Malamud (1914-1986), et plus particulièrement à son roman The Tenants (1971). Les titres des quatre sous-sections dont il se compose sont «The Tenants et la question des fins promises » (p. 55-57), «Le roman sans issue, l'art de tourner en rond» (p.57-60), «La cohabitation impossible» (p.61-63), «Le livre à venir ou l'écriture civilisée» (p.64-67). L'auteur y souligne la tension entre réalisme et métafiction qui est source d'un constant flottement de perspective dans le roman, pour conclure que "l'écriture de Bernard Malamud, avec ses ambiguïtés, ses paradoxes, et ses réticences nous contraint à penser simultanément l'identité et l'altérité, la vérité et le questionnement. Car c'est à cette condition que la Terre Promise (Promised End / Promised Land) se laissera entrevoir, à cette condition que pourra être préservé le texte, si fragile, de notre humanité » (p. 66).

Intitulé «le Masque et la plume», le troisième chapitre de l'ouvrage (p.69-83) est consacré à Philip Roth (1933-), dont The Ghost Writer (1979), premier volume de la tétralogie Zuckerman Bound (1979-1985) est étudié dans quatre sous-sections : "The Ghost Writer: l'écrivain et ses doubles» (p. 72-74), «Une conscience divisée » (p. 74-77), «Anne Frank ressuscitée : la filiation de la réconciliation» (p. 78-79) et «Ecriture et mascarade » (p. 80-83).

Si l'art n'est plus conçu comme la référence suprême, la source ultime de cohérence, il autorise néanmoins un dialogue constructif entre le passé et le présent, entre le Même et l'Autre. Il permet à l'écrivain d'exorciser ses fantômes historiques, littéraires ou personnels, de reconnaître et de célébrer l'inépuisable diversité de l'existence. Car pour Philip Roth, en définitive, la rage d'écrire c'est, d'abord et avant tout, celle de vivre. (p. 83)

Intitulé « la Fascination de l'interdit » le quatrième chapitre (p. 87-111) est consacré à Cynthia Ozick (1935-) et éclaire, entre autres, une interrogation d'ordre religieux, récurrente dans son œuvre, la réconciliation de l'énergie ou de l'impulsion créatrice avec une tradition qui proscrit la représentation : «comment soustraire l'œuvre d'art au sceau de l'interdit?» (p. 88). La fascination qu'exerce l'interdit sur cet écrivain est explorée dans trois nouvelles : "Usurpation (Other People's Stories) », «Puttermesser Her Work History, Her Ancestry, Her Afterlife » et « Puttermesser and Xanthippe », les 
deux dernières constituant un diptyque. L'étude se divise en deux sections dont la première, intitulée "Les 'histoires des autres' » (p. 89-100) se décline en quatre soussections: "Les 'histoires des autres' " (p. 89-91), "Le procès de l'imagination créatrice» (p. 92-95), «Multiples antagonismes» (p. 95-97) et «Un 'miracle' Juif: la couronne rédemptrice » (p. 97-100). La deuxième est intitulée «Le génie de la lettre » (p. 100-112) et contient trois sous-sections: "La 'biographie' de Puttermesser: trahisons de la lettre» (p. 100-103), "Puttermesser et son golem: la lettre prend corps » (104-107), «Inscription et paradoxes : le retrait créateur » (p. 107-112). Dans la première section, le thème central de la nouvelle est dégagé par le biais d'une citation : "The worry is this : whether Jews ought to be story tellers [...] There is only One God and the Muses are not Jewish but Greek» (p. 91). Le récit avance grâce à nombre de ruptures qui aboutissent à la création d'un effet comique illustrant à la fois l'urgence et l'impossibilité du retour aux sources, un retour qui, pour le Juif acculturé, ne peut se faire que sur le mode de l'imaginaire. L'étude se termine avec une citation de MarcAlain Ouaknin commentée par Paule Lévy :

[...] Le messianisme n'est pas la présence du Messie, mais [...] une tension entre le présent et le «à être » du futur [...] D'une certaine manière un peu paradoxale, on peut dire que «le Messie est fait pour ne pas venir ». Le Messie est « à-venir ».

«Selon le judaïsme, commente Paule Lévy, il n'est aucun retour possible à l'harmonie et à l'unité originelles. [...] Le texte de Cynthia Ozick ne cesse de tourner autour de cette béance originelle où il finit par puiser la force même de son élan » (p. 109).

Intitulé "Rires et chuchotements », le cinquième chapitre (p.113-130) est consacré à Grace Paley (1922-2007) et à l'étude de deux nouvelles: "A Conversation with my Father " et "Mother ». "Anti-symbolique » et "anti-mystique ", l'œuvre de Grace Paley réhabilite le banal et donne une visibilité aux démunis et aux faibles (p. 117) tout en renouvelant «en profondeur l'art de la nouvelle» (p.114). Ses nouvelles sont représentatives de deux principaux versants de son œuvre, l'un espiègle, provocateur et ludique, l'autre secret, réservé et intensément poétique. (p. 115)

Intitulé "la Trouée du silence ", le sixième chapitre (p.131-146) est consacré à Tillie Olsen (1912-2007), écrivain moins connue en France que les précédents, et à l'analyse de « Tell me a Riddle». Construite sur une rhétorique du fragment, cette nouvelle met en scène un personnage féminin privé de toute réelle possibilité d'expression ou d'action parce qu'elle ne maîtrise pas la langue du pays où elle se trouve et en rejette les valeurs, arrachée au seul espace qu'elle sentait le sien : sa maison. Dans ce chapitre, Paul Lévy explore le rapport entre littérature et musique.

17 Intitulé "l'Art de dire l'indicible ", le cinquième et dernier chapitre de l'ouvrage (p. 147-164) est consacré à Henry Roth (1906-1995) et plus particulièrement au roman Mercy of a Rude Stream (1994-1997) que l'auteur a composé « au bord de la tombe », après près de soixante années de silence. Le chapitre se clôt avec une citation de Maurice Blanchot (de le Pas au-delà) : "à celui qui a posé tant de questions, la mort vient doucement, comme une question perdue.»

Nombre de citations qui entament, encadrent, étayent ou concluent une réflexion ajoutent, tout au long de l'ouvrage, une qualité poétique et philosophique à la qualité scientifique du propos. En voici quelques exemples:

"Imagination, imagination, imagination! it converts to actual. It sustains, it alters, it redeems." (Saul Bellow) 
«La vie est une perpétuelle distraction qui ne laisse même pas prendre conscience de ce dont elle se distrait. » (Frantz Kafka)

«La judéité, c'est ce qui me manque et non ce qui me définit » (Alain Finkielkraut)

«Tu cherches à te libérer par l'écriture. Quelle erreur! Chaque vocable est le voile soulevé d'un nouveau lien. » (Edmond Jabès)

"Jamais l'esprit ne donne congé à la lettre qui le révèle. Bien au contraire, l'esprit

éveille dans la lettre de nouvelles possibilités de suggestion ». (Emmanuel Lévinas)

"Fiction is getting the world to talk to you." (Grace Paley)

«La musique ne signifie rien mais l'homme qui chante est le lieu de rencontre des significations ». (Vladimir Jankélévitch)

"[...] a life of writing books is a trying adventure in which you cannot find out where you are unless you lose your way." (Philip Roth)

Paule Lévy attache de l'importance à la qualité poétique de sa propre écriture. Cette qualité n'entrave jamais l'accès au sens. Si le propos de Paule Lévy est complexe, les lectures critiques qui l'illustrent sont limpides. Son ouvrage est marqué par une remarquable unité de style et de sens. Dans sa conclusion, Paule Lévy relève ce qui lie les œuvres de son corpus et leurs auteurs, c'est leur dimension éthique, l'humour, ainsi que le concept, bien américain, de «la seconde chance ». Les questions deviennent le socle idéologique et moral de cette littérature dont la force s'affirme par l'acception de la difficulté à avancer des réponses. Laissées ouvertes par les auteurs du corpus, les questions posées dans l'introduction semblent devoir le rester pour le lecteur de l'ouvrage aussi. Néanmoins, sa connaissance de la littérature juive américaine est considérablement affinée puisqu'il a compris que, pour cette littérature, l'assurance que peut offrir l'incarnation est à-venir.

La «bibliographie sélective » qui vient compléter le parcours théorique et critique donne lieu à trois cent trente-deux entrées (p. 175-189) où " seuls sont mentionnés les ouvrages et articles cités dans le volume»(p.175). Elle se divise en trois parties: œuvres littéraires; études critiques sur les littératures américaine, juive et juiveaméricaine ; ouvrages ou articles à caractère théorique (théorie littéraire, philosophie, psychanalyse, religion, histoire).

Cet ouvrage peut se lire sous plusieurs angles: théorique tout d'abord mais aussi "pratique », dans un jeu d'échos entre les nombreuses micro-lectures des extraits cités. Il expose des logiques discursives identitaires, socioculturelles ainsi que la complexification croissante d'un discours sur la construction identitaire de l'artiste et sa représentation à la mesure d'une compréhension plus fine de la pluralité " américaine » au sein de l'identité juive qui est, elle aussi, plurielle dans son unité-on serait tenté de dire " par définition ».

\section{NOTES}

1. Les deux premiers étant Sexualité et textualité dans la littérature américaine contemporaine (1998), ouvrage dirigé par Yves-Charles Grandjeat lui-même et L'Objet et son double: Une relecture de Fizgerald (2008) par Pascal Antolin-Pirès. Un quatrième volume est paru après la sortie de 
l'ouvrage de Paule Lévy, en 2009 : Lionel Larre. Autobiographie amérindienne. Pouvoir et résistance de l'écriture de soi.

2. Par Irving Howe, cité par David Herman dans «Closing Time » dans The Jewish Quarterly, vol. 50, 4, Winter 2003-2004, p. 53-60.

3. Citation de Adrienne Rich, What is to Found there: Notebooks on Poetry and Politics, New York, Norton, 1993, p. 23.

4. Une, deux ou trois œuvres par auteur sont étudiées, selon les cas.

INDEX

Thèmes : Recensions

\section{AUTEUR}

\section{EMMANUEL VERNADAKIS}

Université d'Angers 